\title{
Epigenetics and Its Role in Periodontal Diseases: A State-of-the-Art Review
}

\author{
Lena Larsson, ${ }^{*}$ Rogerio M. Castilho, ${ }^{\dagger}$ and William V. Giannobile ${ }^{\ddagger}$
}

The immune response to oral bacteria and the subsequent activation of inflammatory signaling is not only dependent on genetic factors. The importance of so-called epigenetic mechanisms presents additional regulatory pathways of genes involved in maintaining chronic inflammation, including gingivitis and periodontitis. The term epigenetics relates to changes in gene expression that are not encoded in the DNA sequence itself and include chemical alterations of DNA and its associated proteins. These changes lead to remodeling of the chromatin and subsequent activation or inactivation of a gene. Epigenetic mechanisms have been found to contribute to disease, including cancer and autoimmune or inflammatory diseases. In this state-of-the art review, the authors provide the latest findings on the involvement of epigenetic modifications in the development of periodontal disease and present emerging therapeutic strategies aimed at epigenetic targets (epidrugs) associated with the disruption of tissue homeostasis and the development of periodontitis. J Periodontol 2015;86:556-568.

\section{KEY WORDS}

Acetylation; DNA methylation; epigenomics; genetics; histones; periodontitis.

\footnotetext{
* Currently, Department of Periodontology, Institute of Odontology, University of Gothenburg, Sweden; previously, Department of Periodontics and Oral Medicine, School of Dentistry, University of Michigan, Ann Arbor, MI

$\dagger$ Laboratory of Epithelial Biology, Department of Periodontics and Oral Medicine, School of Dentistry, University of Michigan.

‡ Department of Biomedical Engineering, College of Engineering, University of Michigan. $\S$ Department of Periodontics and Oral Medicine, School of Dentistry, University of Michigan.
}

$\mathrm{P}$ eriodontitis is an infectious disease characterized by chronic inflammation in the gingival tissues in response to bacteria colonizing the tooth surface, ultimately leading to tissue breakdown with loss of alveolar bone and connective tissue. ${ }^{1}$ The epithelial cells in the oral cavity are constantly being exposed to high levels of various bacteria, which leads to activation of multiple signaling pathways that induce various inflammatory response mechanisms. ${ }^{1}$ The host inflammatory response to bacteria is in turn influenced by both genetic and environmental factors. ${ }^{1,2}$

\section{REGULATION OF IMMUNE RESPONSE}

The central dogma of gene expression is that DNA is transcribed into messenger RNA (mRNA), which serves as a template for the protein synthesis. ${ }^{3}$ The basic model for the transcriptional induction in the immune response is that external stimuli, such as bacterial products, start a signaling cascade within the cells, activating the binding of transcription factors to regulatory sequences of the DNA, so-called promoters and enhancers. This assembles the transcription initiation machinery and induces the expression of certain gene sets. ${ }^{4}$ At this level, genetic changes such as mutations, polymorphisms, or alterations in the DNA sequence alter the binding sites for transcription factors in the DNA regulatory region (promoter), thereby affecting 
not only the initiation speed but also the magnitude of the gene expression. In addition, the form of the protein, should these changes occur within the coding regions of the gene (exons), results in changes in the amino acid sequence of the protein. ${ }^{5}$

A recent study has revealed additional regulatory layers superimposed on this basic model. ${ }^{4}$ Specific functional programs able to be rapidly turned on and off in response to external stimuli have been identified. ${ }^{4,6}$ These processes have been proposed as a mechanism in creating a selectivity of the host response to stimuli, and also to regulate the magnitude of the inflammatory response to clear the pathogens or drive chronic inflammation. ${ }^{7}$ In addition, this presents a potential mechanism of how different bacterial species lead to activation of signaling pathways, inducing inflammatory response mechanisms. ${ }^{8}$

The layers of gene expression regulation of an immune response include structures of the chromatin, transcription factors, and posttranscriptional modifications, e.g., RNA splicing, mRNA polyadenylation, and regulatory molecules, which include microRNA (miRNA) and the recently identified long non-coding RNAs (IncRNAs) ${ }^{9}$ (Fig. 1). The first two mechanisms are: 1) modifications of the mRNA transcript and 2) regulation of the stability, storage, and amount of mRNA transcripts present in the cytoplasm. When DNA is transcribed into mRNA, a process called splicing removes the non-coding sequences of mRNA (introns). Bacterial products can alter the splicing pattern, resulting in different isoforms of the protein produced. After the transcription and splicing, the mRNA transcript acquires a sequence of adenosine bases at the terminal end of the transcript called a poly (A)tail. The length of this poly(A) tail determines and contributes to both mRNA stability and transport to the cytoplasm. It also acts as a mechanism for the cell to store silenced mRNA within the cytoplasm, which in response to bacterial stimuli can be used to rapidly induce gene expression without having to start the DNA transcription process in the nucleus. This was also suggested as a way to limit immune response to avoid inducing chronic inflammation. ${ }^{10}$

Not all RNA is protein coding. miRNAs are small, evolutionarily conserved ncRNAs that are derived from longer transcripts encoded within the genome. They inhibit gene expression by binding to mRNA by a sequencepairing homology to regulate and fine-tune gene expression. miRNA expression is altered in response to bacterial products, but inflammatory cytokines can also influence the regulation of

\section{Figure I.}

Epigenetic studies have identified multiple layers of regulatory factors and mechanisms that add to the basic model for transcriptional induction in the immune response. Bacterial products and the inflammatory process influence the transcriptional cascade at all levels. 
miRNA production. ${ }^{7}$ Recently, another class of ncRNAs was discovered, the IncRNAs. ${ }^{7}$ Different biologic functions have been attributed to IncRNAs. A potential mechanism for their ability to regulate gene expression is that they bind to chromatin regulatory proteins, thereby controlling access or inhibition of proteins binding to enhancer regions in the DNA. ${ }^{3,11,12}$ One function of these RNAs in the immune response has been suggested to involve the regulation of the host response, including innate immunity, since virus infection alters the expression of IncRNAs. ${ }^{13}$ Interestingly, a recent report identified a new ncRNA that is linked to epigenetic mechanisms. ${ }^{14}$

Chronic inflammatory diseases, such as periodontitis, have specific target tissues in which the inflammation results in tissue breakdown. ${ }^{1}$ A specific gene may possess different epigenetic patterns de- pending on the cell type, resulting in local and systemic expression of the gene. This indicates a local, site-specific change in the immune response to external stimuli and/or the inflammatory response itself, which may differ among individuals and add to the susceptibility to disease. This review focuses on the epigenetic alterations of the chromatin and DNA, how they can contribute to periodontal disease, and the recent literature on their potential use as new treatment targets.

\section{EPIGENETICS}

The term epigenetics relates to changes in gene expression that are not encoded in the DNA sequence. ${ }^{15}$ Epigenetic modifications include chemical alterations of DNA and associated proteins, leading to remodeling of the chromatin and activation or inactivation of a gene. These changes can contribute

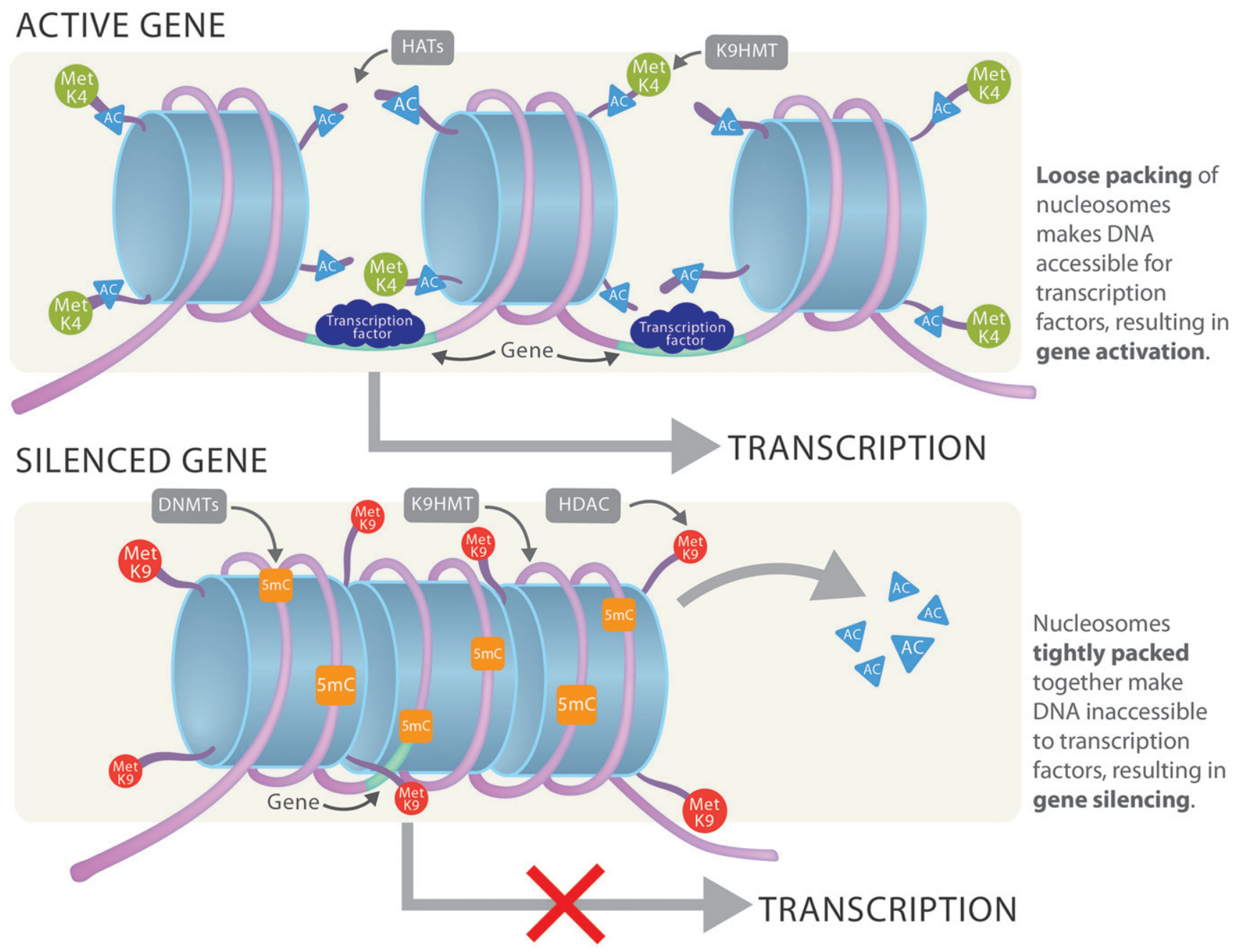

Figure 2.

Schematic illustration of the influence of histone acetylation on gene transcription. Met $=$ methylated; DNMTs $=$ DNA methyltransferases; $5 \mathrm{mC}=$ 5-methylcytosine, $\mathrm{K} 9 \mathrm{HMT}=$ Lysine 9 histone methyltransferase; $\mathrm{AC}=$ acetylation. 
to the development and maintenance of cancer and autoimmune or inflammatory diseases, including periodontitis. Interestingly, some epigenetic modifications are reversible and can be induced and/or altered by environmental factors, therefore presenting a link between the inherited genome and the environment. ${ }^{16,17}$ The two major epigenetic modifications are DNA methylation and histone acetylation and methylation. ${ }^{17}$

The building blocks of chromatin are the nucleosomes. ${ }^{17}$ One nucleosome consists of 146 base pair (bp) DNA and a core histone complex, which includes two copies each of histones H2A, H2B, H3, and H4 and the linker histone $\mathrm{H} 1$ that connects the nucleosomes, forming the primary chromatin structure, often referred to as "beads-on-a-string." Histones can be acetylated or methylated at histone amino acid tails that protrude from the nucleosome. ${ }^{18,19}$ Histone acetylation is regulated by histone acetyltransferases (HATs) and histone deacetylases (HDACs). ${ }^{20} \mathrm{Re}-$ moval of acetyl groups by HDACs leads to alterations in the packing of DNA around histones and a subsequent inactivation of genes (Fig. 2). In contrast, hyperacetylation is associated with transcriptionally active chromatin. ${ }^{20,21}$ Methylation occurs at different lysine molecules on histones $\mathrm{H} 3$ and H4, with $\mathrm{H} 3$ lysines $\mathrm{K} 4$ and $\mathrm{K} 9$ being most commonly methylated. ${ }^{19}$ Although high levels of methylated K4 have been associated with transcriptionally active regions, methylated $\mathrm{K} 9$ was found in silent chro-

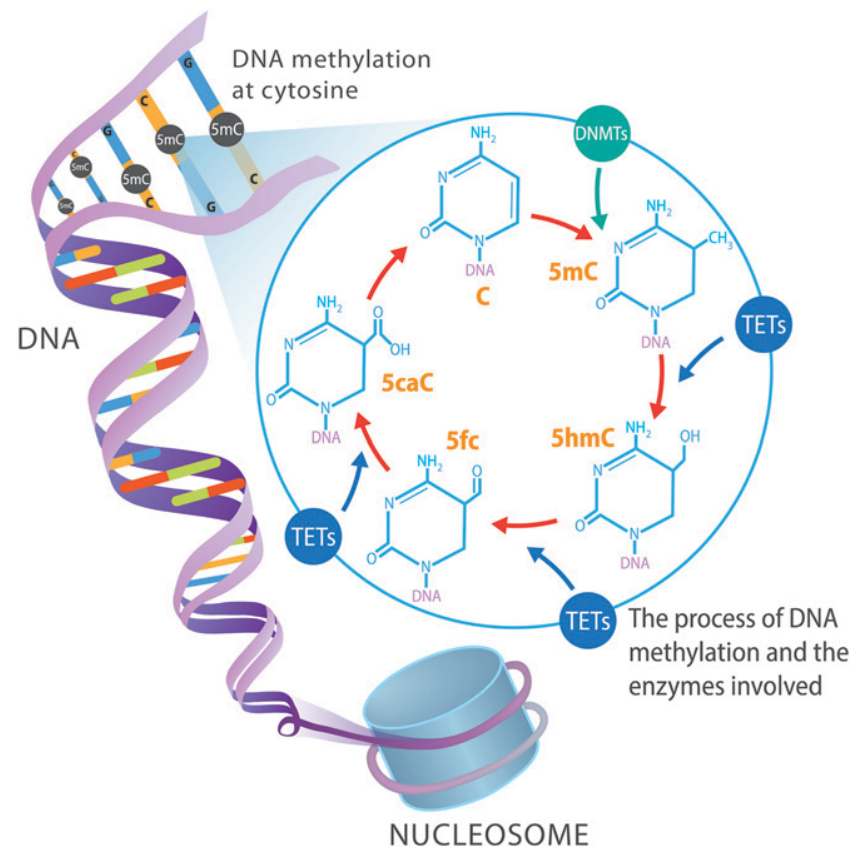

Figure 3.

Illustration of the different forms of DNA methylation and the proteins regulating this process. $5 \mathrm{caC}=5$-carboxydeoxycytidine, $5 f \mathrm{c}=$ 5-formyldeoxycytidine, $\mathrm{c}=$ cytosine. matin regions. ${ }^{19}$ Importantly, a lysine can be mono-, di-, or tri-methylated, further adding to the various functions of methylated histones. ${ }^{19}$ However, recent research in the area of histone methylation reveals evidence for an even more complicated regulation and function of histone methylation, with specific methylation patterns in promoters, introns, and exons resulting in a fine-tuning of gene expression. ${ }^{22}$

In the classic DNA methylation model, the addition of methyl groups to cytosine bases situated next to a guanine base occurs at specific sites in the DNA sequence, so-called $\mathrm{CpG}$ islands or $\mathrm{CpG}$ sites by the de novo DNA methyltransferases (DNMTs) DNMT3a and DNMT3b. ${ }^{15,23}$ This results in both methylated as well as unmethylated CPG sites in the DNA strands. The maintenance enzyme DNMT1 then remethylates hemimethylated $\mathrm{CpG}$ sites. ${ }^{24}$ This modification alters the configuration of the DNA and the binding of transcription factors, resulting in changes in gene expression. Transcriptionally active genes are associated with low levels of DNA methylation. However, in 2014, Jeltsch and Jurkowska ${ }^{24}$ presented a modified version of this classic model based on recent research findings. It was suggested that the average DNA methylation (global DNA methylation) level of DNA is inherited rather than the methylation state of individual CPG sites (site-specific DNA methylation). To add another level to the concept of DNA methylation, it was discovered that 5methylcytosine $(5 \mathrm{mC})$ could be further oxidized into 5 -hydroxymethylcytosine $(5 \mathrm{hmC})$ by the ten-eleven translocation (TET) family of enzymes. ${ }^{25}$ The TET enzymes can then convert $5 \mathrm{hmC}$ into unmethylated cytosine, resulting in DNA hypomethylation. ${ }^{26}$ The biologic function of $5 \mathrm{hmC}$ is not clear, but it has been suggested to be an intermediate leading to demethylation of $5 \mathrm{mC}$ and thereby re-expression of genes silenced by DNA methylation (Fig. 3). ${ }^{27}$

Histone modifications and DNA methylation are not separate events but are linked, and the resulting combinations promote unique cellular responses. ${ }^{21,23}$ In addition, hypo-acetylated histones and hypermethylated CpGs in chronic inflammatory diseases may result in persistent inflammation. ${ }^{20}$ In a recent study, treatment of mice with a compound capable of inducing a immune response (antigen stimulation) resulted in a decrease of DNMT1 followed by an increase in HDAC3. ${ }^{28}$

These changes may provide evidence of the individual differences in local expression of genes associated with inflammation. Specific factors have been reported as risk factors for periodontitis, e.g., smoking, age, bacteria, and genetic polymorphisms. Association between age and risk of developing periodontitis has been reported. ${ }^{29,30}$ Comparison of epigenetic patterns over time in twins at different 


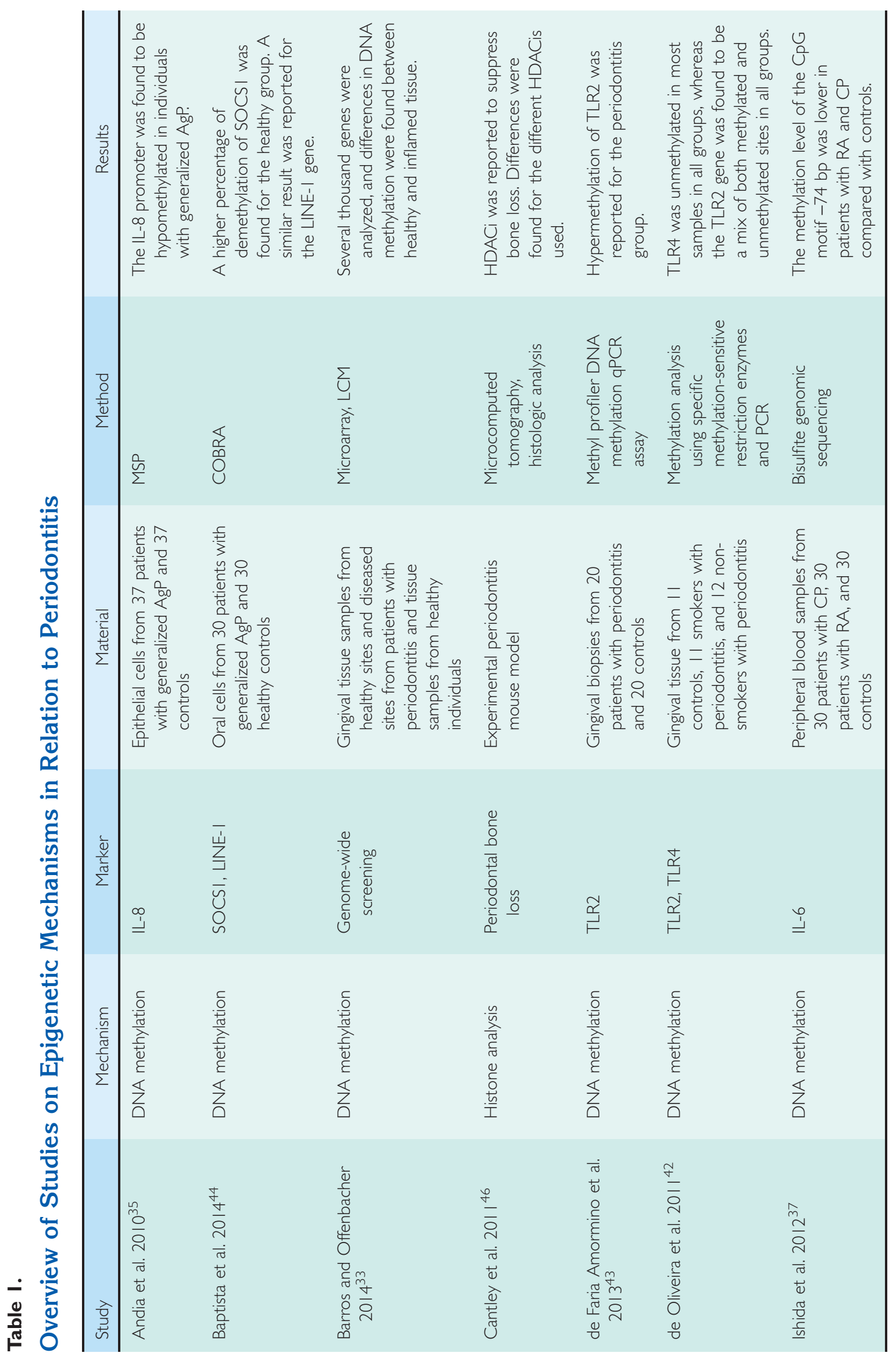




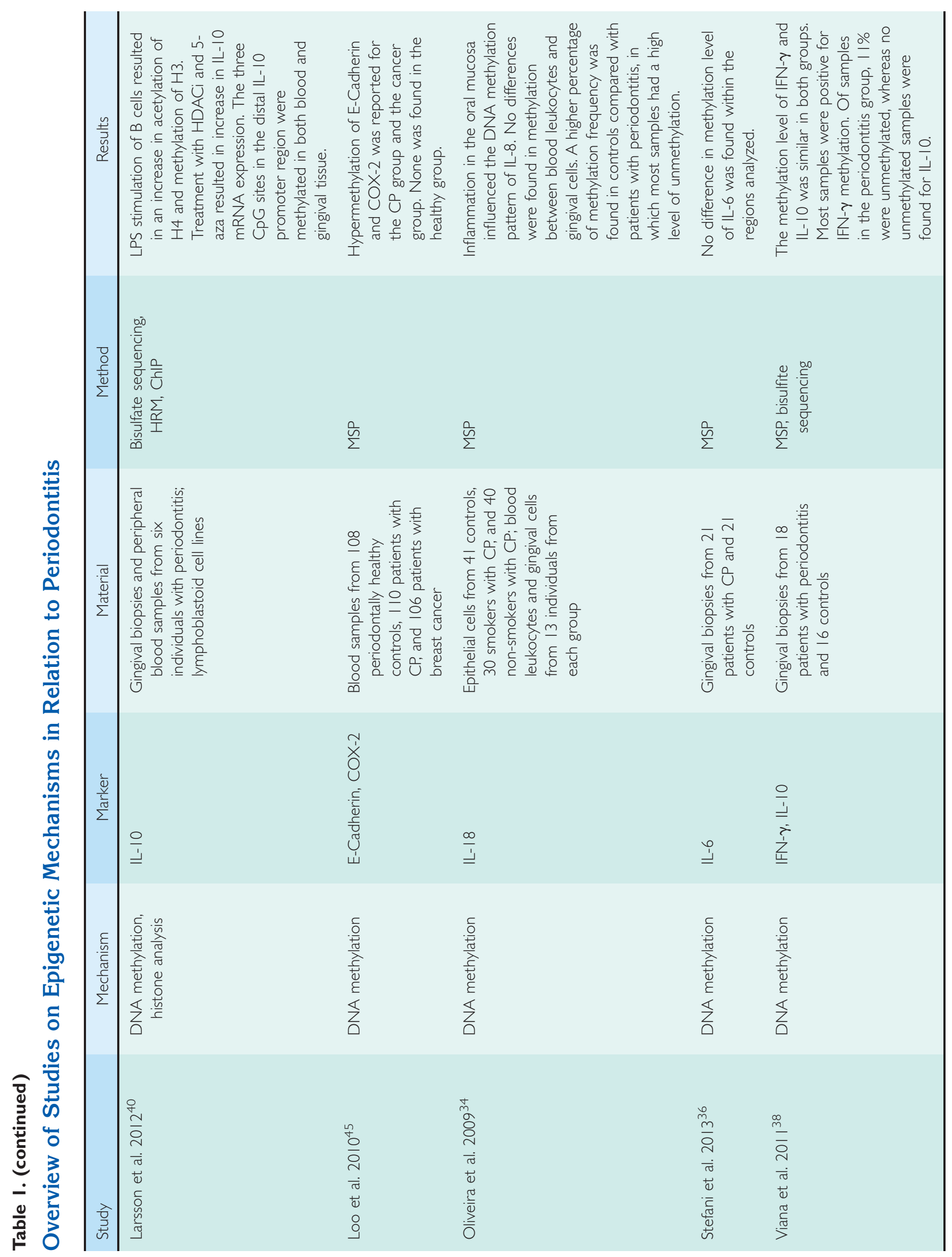




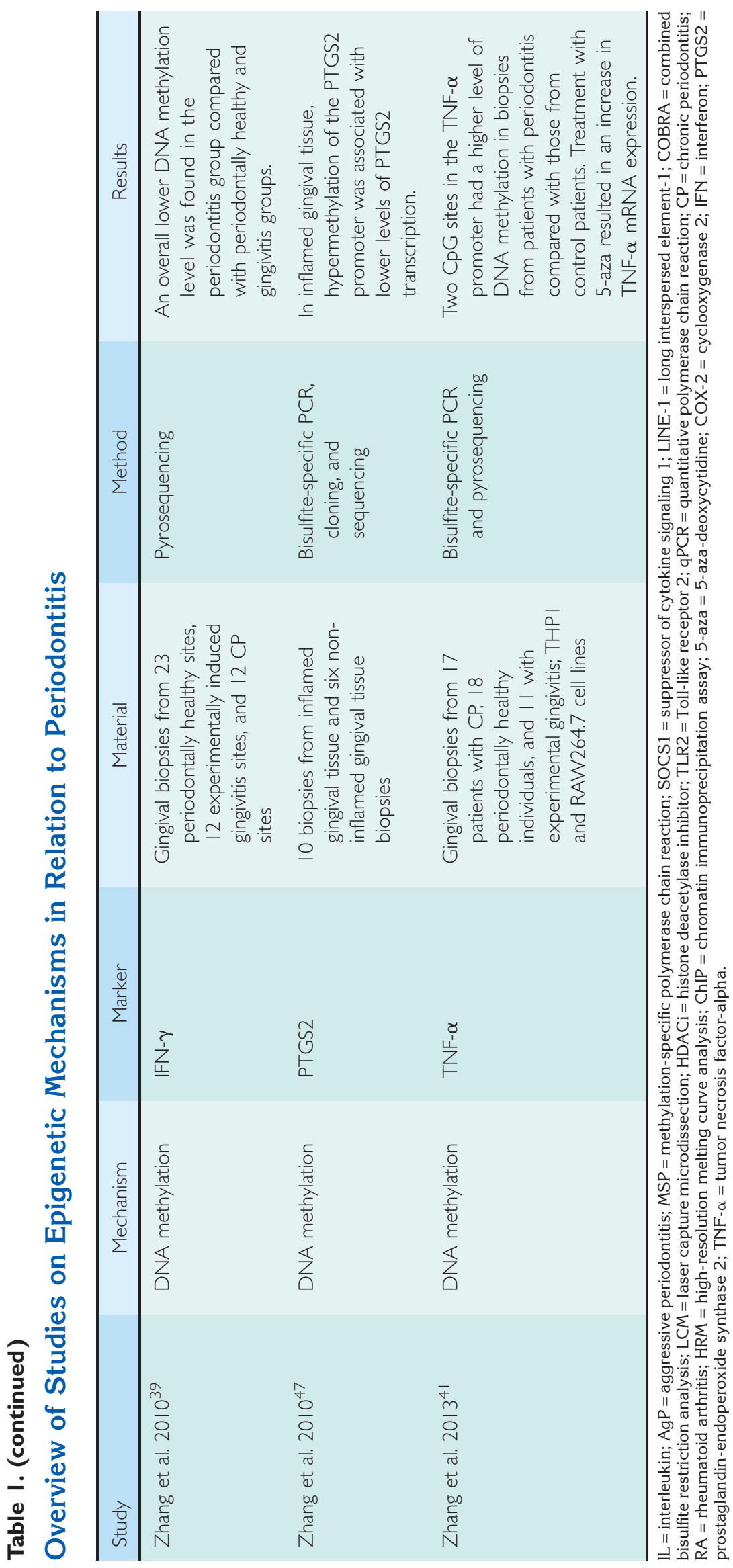

ages showed large differences in gene expression patterns, indicating that epigenetic changes increase during life. However, age itself contrasts with the genetic changes seen in twins, since age itself is a risk factor for epigenetic changes. ${ }^{31}$ In addition, it has been suggested that the epigenome of older patients with periodontitis may differ from that of younger patients. ${ }^{32}$ Epigenetic mechanisms may also explain why some patients may not respond to treatment and why some individuals are more susceptible to periodontitis. ${ }^{32}$ Studies on epigenetic mechanisms in relation to periodontitis ${ }^{33-47}$ are presented in Table 1.

\section{DNA Methylation and Periodontitis}

In periodontitis, epigenetic modifications during inflammation occur locally at the biofilm-gingival interface around the teeth. It has been reported that the epigenome differs between inflamed periodontal sites and non-inflamed sites in the same individual. ${ }^{33}$ In addition, different methylation patterns associated with pathways regulating cell differentiation, apoptosis, lipopolysaccharide (LPS)-mediated signaling, oncogenesis, and cell adhesion were found in inflamed tissue from patients with periodontitis compared with tissue from healthy individuals. ${ }^{33}$

Several studies have investigated the DNA methylation of inflammatory cytokines in chronic periodontitis (CP) and aggressive periodontitis (AgP).34-41 Analysis of the interleukin (IL)-8 promoter indicates a tissue-specific pattern in DNA methylation. ${ }^{34}$ In epithelial cells, the methylation frequency of $I L-8$ was higher in controls than in patients with periodontitis. No difference was seen in gingival cells or blood leukocytes. ${ }^{34}$ These findings are in line with data that found that the IL-8 promoter was hypomethylated in oral epithelial cells from individuals with generalized AgP compared with healthy controls. ${ }^{35}$ The IL- 6 promoter was found to be partially methylated in both healthy individuals and patients with periodontitis, ${ }^{36}$ even though the level of expression of IL-6 was higher in patients with periodontitis. A similar 
increase in IL-6 serum level in patients with CP compared with healthy individuals was associated with hypomethylation of the $-74 \mathrm{bp} \mathrm{CpG}$ site in the IL-6 promoter. This suggests that DNA methylation of a single CPG site may influence cytokine production and hence disease. ${ }^{37}$

The methylation level of interferon (IFN)- $\gamma$ has been investigated in two studies. ${ }^{38,39}$ Viana et al. ${ }^{38}$ reported a similar methylation level of IFN- $\gamma$ and IL-10 in gingival biopsies from patients with $\mathrm{CP}$ compared with biopsies from healthy controls. In contrast, Zhang et al. ${ }^{39}$ reported a lower methylation level of CpG sites in periodontitis biopsies compared with periodontally healthy tissue biopsies. This was related to an increase in IFN- $\gamma$ transcription. The increase in IFN- $\gamma$ in experimental gingivitis was found to be independent of promoter methylation. Regarding the $I L-10$ gene, no unmethylated samples were found in either periodontitis or healthy tissues. ${ }^{38}$ Three $\mathrm{CpG}$ sites close to the IL-10 promoter region around the $-1,087$ polymorphism were methylated in DNA from peripheral blood cells and from gingival tissue. Treatment of $B$ cells with the DNA methylation inhibitor 5-aza-deoxycytidine resulted in an increase in IL-10 mRNA regardless of genotype for the $-1,087$ polymorphism. ${ }^{40}$ Two CpG sites in the tumor necrosis factor (TNF)- $\alpha$ promoter were reported to be hypermethylated in CP. In addition, transcription of TNF - $\alpha$ was shown to be regulated by the methylation level, since treatment of ThP1 cells with 5aza-deoxycytidine increased TNF- $\alpha$ expression. ${ }^{41}$

In addition to methylation level of cytokines, a number of other genes related to inflammation have been analyzed in periodontitis. ${ }^{42-45}$ The DNA methylation pattern in the Toll-like receptor 2 (TLR2) and TLR4 genes was evaluated in gingival tissue samples. ${ }^{42}$ The TLR4 gene promoter was unmethylated in most samples in all groups, ${ }^{42}$ whereas the TLR2 promoter was both methylated and unmethylated in most samples. A hypermethylation and a subsequent low transcription of TLR2 in periodontitis tissues was reported. ${ }^{43}$ Interestingly, a positive correlation was found between TLR2 methylation and periodontal probing depth. In a study on AgP, an overall demethylation pattern of the suppressor of cytokine signaling 1 (SOCS1) gene was found. ${ }^{44}$ In healthy individuals, this demethylated level was higher, and also total demethylated samples were found for this group compared to periodontitis. A significant difference was also reported regarding the methylation pattern for the long interspersed element-1 (LINE-1) gene. ${ }^{44}$

The methylation status of the E-Cadherin and cyclooxygenase 2 (COX-2) genes was investigated in patients with $\mathrm{CP}$ and patients with breast cancer. ${ }^{45}$ The results showed a hypermethylation of these two genes in both patients with periodontitis and those with cancer compared with healthy individuals. These findings confirm the present view that chronic inflammation and cancer may have a similar epigenetic pattern and that DNA methylation may be a link between inflammation and cancer. ${ }^{48}$

A mechanism of how DNA methylation of a gene promoter may influence gene expression was illustrated in a study by Zhang et al. ${ }^{47}$ in which hypermethylation of the prostaglandin-endoperoxide synthase 2 (PTGS2) promoter in inflamed periodontal tissue was associated with lower levels of PTGS2 transcription. Interestingly, a CpG site close to a nuclear factor (NF)-кB transcription factor-binding site was highly methylated, suggesting that methylation status may exclude binding of this transcription factor. This is in line with other studies on methylation of cytokine promoter regions in which the CPG sites analyzed were in close proximity to binding sites for transcription factors $\mathrm{NF}-\kappa \mathrm{B}$ and specificity protein 1 (Sp1). ${ }^{40,41}$ These two factors are known to be strong regulators of gene expression of the immune response. They are activated by bacteria binding to TLRs and the subsequent activation of mitogenactivated protein kinase (MAPK) pathways. ${ }^{49,50}$ The influence of Sp1 and NF- $\mathrm{KB}$ on the chromatin structure was demonstrated by Widlak et al. in $1997,{ }^{51}$ who showed that the binding of Sp1 and NF-кB to adjacent sites induced a repositioning of the nucleosomes upstream and downstream of the factors, thereby influencing gene transcription. ${ }^{51}$ It has been reported that enzymes that cause methylation can be regulated directly or indirectly by the extracellular milieu through signal transduction pathways such as the MAPK extracellular signal-regulated kinase (ERK) kinase pathway. ${ }^{52}$

The inflammatory processes may also result in aberrant DNA methylation in tissues. ${ }^{53}$ This mechanism has been suggested as a link between inflammation and alterations in methylation level in cancers. In inflamed tissue, neutrophils and eosinophils produce substances that further form halogenated cytosine products. ${ }^{53}$ The DNMT1 enzyme cannot distinguish between halogenated and methylated DNA, resulting in an increase in de novo methylation in cells of the inflamed tissue. ${ }^{53}$ In addition, antigen stimulation can result in downregulation of DNMT1 and HDAC2, with an increase in HDAC3, together with induction of Sp1 and NF-kB p65 binding to the HDAC3 promoter. The downregulation of DNMT1 results in induction of adhesion molecules and cellular interactions. ${ }^{28}$

The process of $5 \mathrm{mC}$ oxidation into $5 \mathrm{hmC}$ has been well studied in cancer, but understanding of the role of these markers in inflammation is limited. In a study on the autoimmune disease systemic lupus erythematosus (SLE), ${ }^{54}$ the levels of TET1, TET2, and $5 \mathrm{hmC}$ in cluster of differentiation 4-positive $\left(\mathrm{CD} 4^{+}\right) \mathrm{T}$ cells were 
compared to those from healthy individuals. A high level of these markers in patients with SLE was found. It was suggested that the DNA hydroxymethylation may be contributing to the pathogenesis of SLE ${ }^{54}$ Because SLE and periodontitis share common features regarding the immune response, it may also be that DNA hydroxymethylation plays a role in periodontitis similar to that in SLE. ${ }^{55}$ Bacterial influence of TET2 expression has been previously shown, as Escherichia coli stimulation of THP1 cells for 24 hours resulted in more than double the amount of TET2 mRNA. ${ }^{56}$

\section{Histone Modifications and Periodontitis}

Few studies are available on histone modifications in periodontitis. ${ }^{40,46}$ Maintaining histone acetylation of genes related to osteoclastogenesis was found to be important for preventing bone loss in experimental periodontitis. Treatment with histone deacetylase inhibitor (HDACi) resulted in improved bone levels. ${ }^{53}$ In a study from the authors' laboratory, ${ }^{40}$ it was demonstrated that different epigenetic modifications of DNA and histones influence IL-10 gene expression. The authors also found an association between LPS stimulation and histone modifications. Furthermore, these alterations differed among IL-10 genotypes for the $-1,087 \mathrm{G} / \mathrm{A}$ polymorphism in the IL-10 gene promoter. ${ }^{40}$ Administration of HDACi trichostatin A did not alter the IL-10 production; however, treatment using HDACi butyrate and valproic acid resulted in an increase in IL-10 gene expression.

\section{Influence of Periodontal Pathogens on Chromatin Modifications}

An early report on the bacterial influence on DNA methylation showed that bacterial infection with Campylobacter rectus promoted DNA hypermethylation of the insulin-like growth factor 2 (IGF2) gene. ${ }^{57}$ Stimulation with the periodontal pathogen Treponema denticola on periodontal ligament cells showed a decrease in methylation of the matrix metalloproteinase (MMP)-2 promoter. ${ }^{58}$ However, the authors could not confirm these find ings using the more sensitive bisulfite DNA sequencing method. ${ }^{58}$ In addition, the MMP-2 promoter was found to be hypomethylated regardless of the presence of $T$. denticola. It was suggested that the adherence and/or internalization of $T$. denticola may contribute to regulation of matrix degradation and bone resorption. ${ }^{58}$ Studies have shown that the culture supernatant from the periodontal pathogen Porphyromonas gingivalis contains high levels of butyric acid. ${ }^{59,60}$ This culture supernatant was reported to inhibit HDACs, resulting in increased histone acetylation. ${ }^{59,60}$

Periodontal pathogens can induce cell-specific changes as shown in a study ${ }^{61}$ comparing the expression of epigenetic markers in gingival fibroblasts and keratinocytes. $P$. gingivalis LPS stimulation re- sulted in a decrease in mRNA transcription of DNMT1, DNMT3, and histone demethylases Jumonji domain containing 3 (JMJD3) in keratinocytes but not in gingival fibroblasts. In contrast, RNA analysis from gingival biopsies from periodontitis and healthy sites showed no difference in gene expression for these target genes. ${ }^{61}$ JMJD3 binds to genes and regulates the H3K27me3 level and hence transcription activity. It was suggested that a decrease in JMJD3 could lead to changes in transcription levels of inflammatory genes activated by LPS. Interestingly, JMJD3 is regulated by NF-кB. ${ }^{61}$ Consistent with these findings are the results presented by Yin and Chung, ${ }^{8}$ who investigated the influence of $P$. gingivalis and Fusobacterium nucleatum on epigenetic modifications in gingival epithelial cells. $P$. gingivalis stimulation resulted in a decrease in HDAC1, HDAC2, and DNMT1, whereas no difference was found after stimulation with $F$. nucleatum for those markers compared with untreated cells. These results indicate that oral bacterial infection is associated with changes in the histone H3 lysine 4 (H3K4) methylation level, and that the bacteria investigated induced different levels of trimethylated H3K4. In addition, these bacteria drove different epigenetic changes for the antimicrobial proteins human $\beta$-defensin 2 and CC chemokine ligand 20.

Specific cells within the oral mucosa may respond differently to bacterial infection or to the periodontal inflammatory processes. ${ }^{34}$ This results in differences in the methylation pattern of a certain gene among cell types. Analysis of epigenetic modifications from gingival biopsies shows the combined results of all cells present in the sample, making it difficult to estimate the role of each cell type in the epigenetic pattern at the site of inflammation. ${ }^{61}$ Barros and Offenbacher used laser capture microdissection (LCM) to analyze epithelial cells from the sulcular epithelium. ${ }^{33}$ A large number of genes were analyzed, but the gamma-amino-n-butyrate transaminase (GATA) gene better illustrated differences in gene expression between the gingival biopsies and LCM tissue samples. The results from gingival samples showed a 2.5-fold increase in GATA gene methylation, comparing healthy individuals versus patients with periodontitis. This was confirmed using LCM, with which a 1.2-fold increase was found.

However, not only do bacteria influence the methylation level of a host's genes, the bacterial DNA can also differ in methylation level, which influences their ability to induce the production of inflammatory cytokines. ${ }^{62}$ The production of TNF- $\alpha$ in macrophages was induced only when bacterial DNA from Aggregatibacter actinomycetemcomitans, $P$. gingivalis, Peptostreptococcus micros, and E. coli was unmethylated. ${ }^{62}$ 
The studies on epigenetics and periodontitis describe how the immune response influences the epigenetic pattern of genes. However, it should be noted that epigenetic changes may also affect the immune response itself, thereby providing a potential explanation for how individuals are more prone to develop chronic inflammation.

\section{CLINICAL APPLICATIONS TARGETING EPIGENETIC MODIFICATIONS}

Limited research has been done using HDAC or DNA methylation inhibitors as treatments for periodontitis or oral inflammation. In contrast, there are numerous reports on the use of these epigenetic inhibitors in cancer research. In a recent review, ${ }^{63}$ different areas for use of epigenetic factors were identified to improve individualized drug therapy, in which pharmacoepigenetic biomarkers can be used to predict drug response. One example is the O6-methylguanine DNA methyltransferase molecule that can be detected in serum and saliva at levels corresponding to that of the primary tumor. In this manner, it would be possible to monitor epigenetic changes in a specific tissue by using more easily accessible body fluids. Epidrugs are defined as "drugs that inhibit or activate disease-associated epigenetic proteins for ameliorating, curing, or preventing the disease." 63 Indeed, the present authors have found that administration of epidrugs (HDACi) can prevent the accumulation of

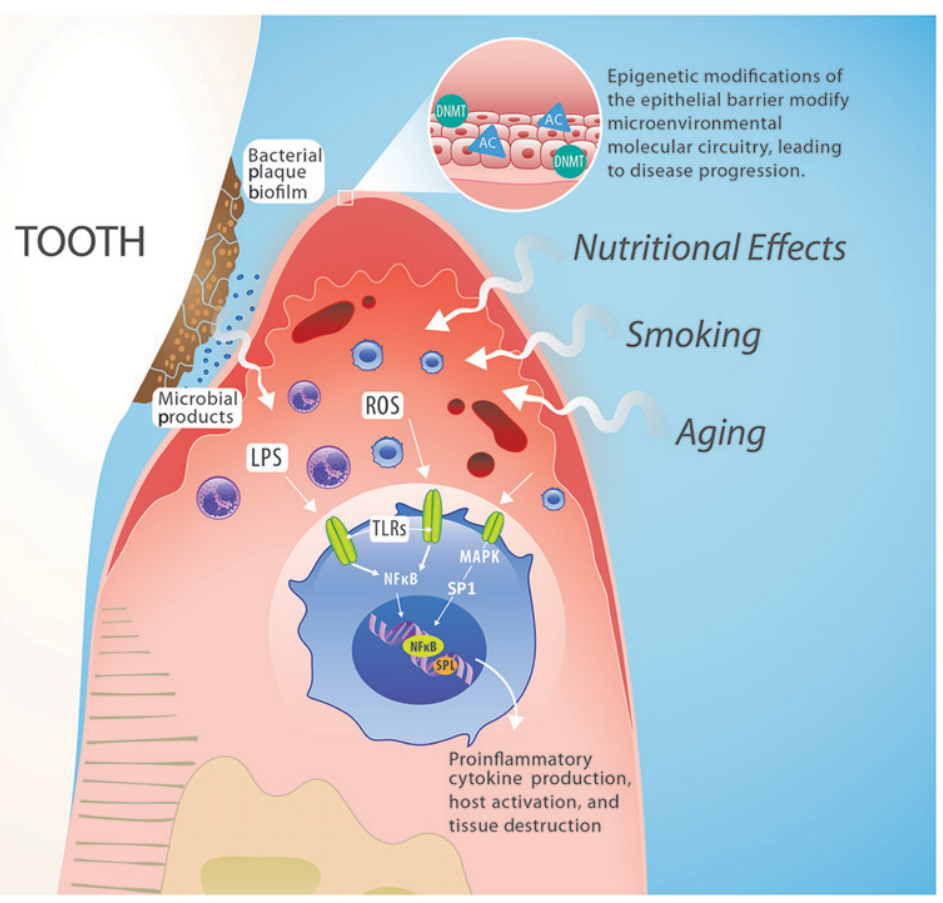

Figure 4.

Based on the studies presented in the present review, a potential hypothesis of the epigenetic events occurring in a CP lesion. $R O S=$ reactive oxygen species. $A C=$ acetylase; $S P I=$ transcription factor. head and neck cancer stem cells and increase the sensitivity of tumor cells to chemotherapy. ${ }^{64,65}$ At present, two DNMT inhibitors and two HDAC inhibitors have been approved by the U.S. Food and Drug Administration. Although these developments are exciting, the complexity of solid tumors are far beyond current understanding, and the influence of gene expression mediated by histone modifications is in part impacted by the latest identification of several mutations in histones and histone modifiers. ${ }^{66}$

Recently, HDACis have been found not only to regulate histone acetylation but also to influence DNA methylation. ${ }^{52}$ The HDACi suberoylanilide hydroxamic acid inhibits histone acetylation but also regulates DNMT1 protein expression. ${ }^{52}$ Treatment using another HDAC inhibitor, 1,179.4b (Classes I and II HDACi), showed reduced alveolar bone loss and less osteoclasts per square millimeter in mice with $P$. gingivalis-induced periodontitis compared with untreated mice. ${ }^{46}$ However, no influence was found on the level of inflammation, which was suggested to indicate a direct effect of this HDACi on bone regeneration rather than an indirect effect by targeting the inflammation. ${ }^{46}$ Moderate daily exercise and diet were found to reduce $P$. gingivalisinduced periodontitis in diet-induced obese mice by restoring the level of histone 3 acetylation to normal, as well as NF- $\kappa B$ recruitment to TNF- $\alpha$ and IL-10 promoter. ${ }^{67}$ It should be noted that $P$. gingivalis produces butyric acid that inhibits HDACs and increases histone acetylation, which was shown to reactivate Epstein-Barr virus (EBV) as well as human immunodeficiency virus 1 , indicating that periodontal disease may contribute to EBV-related diseases. ${ }^{59,60}$

A group of epigenetic regulatory proteins are the bromodomain and extraterminal domain (BET) proteins, which are able to read acetylated histone tails and recruit transcription complexes to regulate gene transcription. In a recent study, the BET inhibitor JQ1 was found to inhibit both an inflammatory response and alveolar bone loss in experimental periodontitis. In vitro analysis of the function of JQ1 revealed that binding of the BET protein BRD4 to the NF- $\mathrm{NB}$ promoters was decreased, as was NF-кB phosphorylation, and treatment with JQ1 also attenuated nuclear NF$\kappa B$. Based on these findings, it 
was suggested that JQ1 may be a potential treatment model for periodontitis. ${ }^{68}$ Interestingly, aspirin has been found to have a negative effect on allergic inflammation by regulating DNMT1 expression. In this model, aspirin was shown to prevent antigen from influencing the expression of HAT Tip60, HDAC3, and DNMT1. ${ }^{28}$

\section{CONCLUSIONS}

Recent studies have reported on the ability and importance of environmental factors to induce changes in epigenetic regulatory mechanisms of the immune response (Fig. 4). Knowledge of the role of epigenetics in development of periodontal diseases is still limited, but the studies presented in this review highlight that both DNA methylation and histone modifications occur in the oral mucosa in response to bacteria and the inflammatory processes. To better understand the influence of environmental cues during the development of periodontal disease, the molecular events driven by epigenetic modifications need to be identified. This information may also provide some explanations as to why patients with similar clinical characteristics respond differently to treatment. Identifying epigenetic patterns associated with the development of periodontitis may also improve personalized approaches that can be used to individualize treatment plans for patients with periodontitis.

\section{ACKNOWLEDGMENTS}

This work was supported by IRIS stipend (Gothenburg, Sweden) to LL, Colgate Palmolive Company (Piscataway, New Jersey) to WVG, and the University of Michigan School of Dentistry (Ann Arbor, Michigan) to RMC. The authors report no conflicts of interest related to this study.

\section{REFERENCES}

1. Kornman KS. Mapping the pathogenesis of periodontitis: A new look. J Periodontol 2008;79(Suppl. 8):1560-1568

2. Offenbacher S, Barros SP, Beck JD. Rethinking periodontal inflammation. J Periodontol 2008;79(Suppl. 8):1577-1584.

3. Rinn JL, Chang HY. Genome regulation by long noncoding RNAs. Annu Rev Biochem 2012;81:145166.

4. Smale ST. Transcriptional regulation in the innate immune system. Curr Opin Immunol 2012;24:51-57.

5. Shastry BS. SNPs: Impact on gene function and phenotype. Methods Mol Biol 2009;578:3-22.

6. Ivanov P, Anderson P. Post-transcriptional regulatory networks in immunity. Immunol Rev 2013;253:253-272.

7. O'Connell RM, Rao DS, Baltimore D. microRNA regulation of inflammatory responses. Annu Rev Immunol 2012;30:295-312.
8. Yin L, Chung WO. Epigenetic regulation of human $\beta$-defensin 2 and CC chemokine ligand 20 expression in gingival epithelial cells in response to oral bacteria. Mucosal Immunol 2011;4:409-419.

9. Carpenter S, Ricci EP, Mercier BC, Moore MJ, Fitzgerald KA. Post-transcriptional regulation of gene expression in innate immunity. Nat Rev Immunol 2014; 14:361-376.

10. Weill L, Belloc E, Bava F-A, Méndez R. Translational control by changes in poly(A) tail length: Recycling mRNAs. Nat Struct Mol Biol 2012;19:577-585.

11. Bonasio R, Shiekhattar R. Regulation of transcription by long noncoding RNAs. Annu Rev Genet 2014;48: 433-455.

12. Zhang X, Li H, Burnett JC, Rossi JJ. The role of antisense long noncoding RNA in small RNA-triggered gene activation. RNA 2014;20:1916-1928.

13. Peng X, Gralinski L, Armour CD, et al. Unique signatures of long noncoding RNA expression in response to virus infection and altered innate immune signaling. MBio 2010;1:e00206.

14. Di Ruscio A, Ebralidze AK, Benoukraf T, et al. DNMT1interacting RNAs block gene-specific DNA methylation. Nature 2013;503:371-376.

15. Bird A. DNA methylation patterns and epigenetic memory. Genes Dev 2002;16:6-21.

16. Barros SP, Offenbacher S. Epigenetics: Connecting environment and genotype to phenotype and disease. $J$ Dent Res 2009;88:400-408.

17. Wilson AG. Epigenetic regulation of gene expression in the inflammatory response and relevance to common diseases. J Periodontol 2008;79(Suppl. 8):1514-1519.

18. Szerlong HJ, Hansen JC. Nucleosome distribution and linker DNA: Connecting nuclear function to dynamic chromatin structure. Biochem Cell Biol 2011;89:24-34.

19. Turner BM. Cellular memory and the histone code. Cell 2002;111:285-291.

20. Bäckdahl L, Bushell A, Beck S. Inflammatory signalling as mediator of epigenetic modulation in tissue-specific chronic inflammation. Int J Biochem Cell Biol 2009;41:176-184.

21. Jenuwein T, Allis CD. Translating the histone code. Science 2001;293:1074-1080.

22. Black JC, Van Rechem C, Whetstine JR. Histone lysine methylation dynamics: Establishment, regulation and biological impact. Mol Cell Rev 2012;48:491-507.

23. Robertson KD, Wolffe AP. DNA methylation in health and disease. Nat Rev Genet 2000;1:11-19.

24. Jeltsch A, Jurkowska RZ. New concepts in DNA methylation. Trends Biochem Sci 2014;39:310-318.

25. Tahiliani M, Koh KP, Shen Y, et al. Conversion of 5 -methylcytosine to 5-hydroxymethylcytosine in mammalian DNA by MLL partner TET1. Science 2009;324: 930-935.

26. Ito $S$, Shen L, Dai $Q$, et al. Tet proteins can convert 5-methylcytosine to 5-formylcytosine and 5-carboxylcytosine. Science 2011;333:1300-1303.

27. Kraus TF, Globisch D, Wagner M, et al. Low values of 5hydroxymethylcytosine $(5 \mathrm{hmC})$, the "sixth base," are associated with anaplasia in human brain tumors. Int $J$ Cancer 2012;131:1577-1590.

28. Kim Y, Kim K, Park D, et al. DNA methyl transferase I acts as a negative regulator of allergic skin inflammation. Mol Immunol 2013;53:1-14.

29. Eke PI, Dye BA, Wei L, Thornton-Evans GO, Genco RJ; CDC Periodontal Disease Surveillance Workgroup. Prevalence of periodontitis in adults in the 
United States: 2009 and 2010. J Dent Res 2012;91: 914-920.

30. Papapanou PN, Lindhe J. Epidemiology of periodontal disease. In: Lindhe J, Lang NP, Karring T, eds. Clinical Periodontology and Implant Dentistry, 5th ed. Oxford: Blackwell Munksgaard; 2008:129-179.

31. Fraga MF, Ballestar E, Paz MF, et al. Epigenetic differences arise during the lifetime of monozygotic twins. Proc Natl Acad Sci USA 2005;102:1060410609.

32. Lod S, Johansson T, Abrahamsson KH, Larsson L. The influence of epigenetics in relation to oral health. Int $J$ Dent Hyg 2014;12:48-54.

33. Barros SP, Offenbacher S. Modifiable risk factors in periodontal disease: Epigenetic regulation of gene expression in the inflammatory response. Periodontol 2000 2014;64:95-110.

34. Oliveira NF, Damm GR, Andia DC, et al. DNA methylation status of the $I L 8$ gene promoter in oral cells of smokers and non-smokers with chronic periodontitis. $J$ Clin Periodontol 2009;36:719-725.

35. Andia DC, de Oliveira NF, Casarin RC, Casati MZ, Line SR, de Souza AP. DNA methylation status of the IL8 gene promoter in aggressive periodontitis. J Periodontol 2010;81:1336-1341.

36. Stefani FA, Viana MB, Dupim AC, et al. Expression, polymorphism and methylation pattern of interleukin-6 in periodontal tissues. Immunobiology 2013;218: 1012-1017.

37. Ishida $\mathrm{K}$, Kobayashi $T$, Ito $S$, et al. Interleukin- 6 gene promoter methylation in rheumatoid arthritis and chronic periodontitis. J Periodontol 2012;83:917-925.

38. Viana MB, Cardoso FP, Diniz MG, et al. Methylation pattern of IFN- $\gamma$ and IL-10 genes in periodontal tissues. Immunobiology 2011;216:936-941.

39. Zhang S, Crivello A, Offenbacher S, Moretti A, Paquette DW, Barros SP. Interferon-gamma promoter hypomethylation and increased expression in chronic periodontitis. J Clin Periodontol 2010;37:953-961.

40. Larsson L, Thorbert-Mros S, Rymo L, Berglundh T. Influence of epigenetic modifications of the interleukin10 promoter on IL10 gene expression. Eur J Oral Sci 2012;120:14-20.

41. Zhang S, Barros SP, Moretti AJ, et al. Epigenetic regulation of TNFA expression in periodontal disease. $J$ Periodontol 2013;84:1606-1616.

42. De Oliveira NF, Andia DC, Planello AC, et al. TLR2 and TLR4 gene promoter methylation status during chronic periodontitis. J Clin Periodontol 2011;38:975-983.

43. de Faria Amormino SA, Arao TC, Saraiva AM, et al. Hypermethylation and low transcription of TLR2 gene in chronic periodontitis. Human Immunobiol 2013;74: 1231-1236.

44. Baptista NB, Portinho D, Casarin RC, et al. DNA methylation levels of SOCS1 and LINE-1 in oral epithelial cells from aggressive periodontitis patients. Arch Oral Biol 2014;59:670-678.

45. Loo WT, Jin L, Cheung MN, Wang M, Chow LW. Epigenetic change in E-cadherin and COX-2 to predict chronic periodontitis. J Transl Med 2010;8:110-115.

46. Cantley MD, Bartold PM, Marino V, et al. Histone deacetylase inhibitors and periodontal bone loss. J Periodontal Res 2011;46:697-703.

47. Zhang S, Barros SP, Niculescu MD, Moretti AJ, Preisser JS, Offenbacher S. Alteration of PTGS2 promoter methylation in chronic periodontitis. J Dent Res 2010; 89:133-137.
48. Kundu JK, Surh YJ. Inflammation: Gearing the journey to cancer. Mutat Res 2008;659:15-30.

49. Saccani S, Pantano S, Natoli G. p38-Dependent marking of inflammatory genes for increased NF-kappa B recruitment. Nat Immunol 2002;3:69-75.

50. Liu Y-W, Chen C-C, Tseng H-P, Chang W-C. Lipopolysaccharide-induced transcriptional activation of interleukin-10 is mediated by MAPK- and NFkappaB-induced CCAAT/enhancer-binding protein $\delta$ in mouse macrophages. Cell Signal 2006;18:14921500.

51. Widlak P, Gaynor RB, Garrard WT. In vitro chromatin assembly of the HIV-1 promoter. ATP-dependent polar repositioning of nucleosomes by Sp1 and NFkappaB. J Biol Chem 1997;272:17654-17661.

52. Sarkar S, Abujamra AL, Loew JE, Forman LW, Perrine SP, Faller DV. Histone deacetylase inhibitors reverse CPG methylation by regulating DNMT1 through ERK signaling. Anticancer Res 2011;31:2723-2732.

53. Valinluck V, Sowers LC. Endogenous cytosine damage products alter the site selectivity of human DNA maintenance methyltransferase DNMT1. Cancer Res 2007;67:946-950.

54. Zhang Y, Zhao M, Sawalha AH, Richardson B, Lu Q. Impaired DNA methylation and its mechanisms in CD4(+) $T$ cells of systemic lupus erythematosus. $J$ Autoimmun 2013;41:92-99.

55. Berglundh T, Donati M, Zitzmann N. B cells in periodontitis: Friends or enemies? Periodontol 2000 2007; 45:51-66.

56. Neves-Costa A, Moita LF. TET1 is a negative transcriptional regulator of IL-1 $\beta$ in the THP-1 cell line. Mol Immunol 2013;54:264-270.

57. Bobetsis YA, Barros SP, Lin DM, et al. Bacterial infection promotes DNA hypermethylation. J Dent Res 2007;86:169-174.

58. Miao D, Godovikova V, Qian X, Seshadrinathan S, Kapila YL, Fenno JC. Treponema denticola upregulates MMP-2 activation in periodontal ligament cells: Interplay between epigenetics and periodontal infection. Arch Oral Biol 2014;59:1056-1064.

59. Imai K, Ochiai K, Okamoto T. Reactivation of latent HIV-1 infection by the periodontopathic bacterium Porphyromonas gingivalis involves histone modification. J Immunol 2009;182:3688-3695.

60. Imai $\mathrm{K}$, Inoue $\mathrm{H}$, Tamura $\mathrm{M}$, et al. The periodontal pathogen Porphyromonas gingivalis induces the Epstein-Barr virus lytic switch transactivator ZEBRA by histone modification. Biochimie 2012;94:839. 846.

61. de Camargo Pereira G, Guimarães GN, Planello AC, et al. Porphyromonas gingivalis LPS stimulation downregulates DNMT1, DNMT3a, and JMJD3 gene expression levels in human HaCaT keratinocytes. Clin Oral Investig 2013;17:1279-1285.

62. Nonnenmacher C, Dalpke A, Zimmermann S, FloresDe-Jacoby L, Mutters R, Heeg K. DNA from periodontopathogenic bacteria is immunostimulatory for mouse and human immune cells. Infect Immun 2003;71:850856.

63. Ivanov M, Barragan I, Ingelman-Sundberg M. Epigenetic mechanisms of importance for drug treatment. Trends Pharmacol Sci 2014;35:384-396.

64. Almeida LO, Abrahao AC, Rosselli-Murai LK, et al. $\mathrm{NF \kappa B}$ mediates cisplatin resistance through histone modifications in head and neck squamous cell carcinoma (HNSCC). FEBS Open Bio 2014;4:96-104. 
65. Giudice FS, Pinto DS Jr., Nör JE, Squarize CH, Castilho RM. Inhibition of histone deacetylase impacts cancer stem cells and induces epithelial-mesenchyme transition of head and neck cancer. PLOS ONE 2013;8: e58672.

66. Martins MD, Castilho RM. Histones: Controlling tumor signaling circuitry. J Carcinog Mutagen 2013;29 (Suppl. 5):1-12.

67. Zhou Q, Leeman SE, Amar S. Signaling mechanisms in the restoration of impaired immune function due to diet-induced obesity. Proc Natl Acad Sci USA 2011; 108:2867-2872.
68. Meng S, Zhang L, Tang Y, et al. BET inhibitor JQ1 blocks inflammation and bone destruction. J Dent Res 2014;93:657-662.

Correspondence: Dr. William V. Giannobile, Department of Periodontics and Oral Medicine, University of Michigan School of Dentistry, 1011 North University, Ann Arbor, MI 48109-1078. Fax: 734/763-5503; e-mail: william.giannobile@umich.edu.

Submitted October 1, 2014; accepted for publication November 12, 2014. 